\title{
Wives' perception of sexual relationship with narcissistic men: Results of a qualitative thematic analysis with Iranian women
}

\author{
Iesa Moradi ${ }^{\circledR}$, Maryam Fatehizade $^{2^{*}(\mathbb{D}}$, Ahmad Ahmadi $^{3}$, Ozra Etemadi ${ }^{4}$ \\ ${ }^{1}$ Ph.D. in Counseling, University of Isfahan, Isfahan, Iran \\ ${ }^{2}$ Associate Professor of Counseling Group, University of Isfahan, Isfahan, Iran \\ ${ }^{3}$ Professor of Counseling Group, University of Isfahan, Isfahan, Iran \\ ${ }^{4}$ Associate Professor of Counseling Group, University of Isfahan, Isfahan, Iran
}

*Corresponding Author: Maryam Fatehizade, Tel: 09131004925, Email: m.fatehizade@edu.ui.ac.ir

\begin{abstract}
Background and aims: Many studies have investigated the interpersonal dimensions of narcissism, though women's perceptions of sexual relationship with a narcissistic individual have rarely been the topic of interest. The main purpose of this study was to investigate the sexual lived experiences of women living with narcissistic men.

Methods: To this end, a qualitative thematic analysis was used to have a better understanding of the concept. A total of 31 women were selected through convenience sampling technique and were interviewed using a semi-structured interview, all of whom were living with their narcissistic husbands. All interviews were recorded, transcribed, and analyzed by thematic analysis.

Results: Based on the findings of this study, two main themes emerged during data analysis. First, women found their sexual relationship with a narcissistic man insecure, as well as the perception that their relationship was one-sided and demanding. In addition, the insecured relationship comprised several subthemes including the lack of sexual intimacy, sexual aggression and unwanted sex, and low commitment. The other main themes contained two sub-themes, namely, unrealistic and excessive expectations from the spouse and the relationship and the lack of responsibility and cooperation of the husband.

Conclusion: This study was mainly focused on sexuality in living with a narcissistic person and the results showed that sex is strongly influenced by the consequences of narcissism. This study also has some research and clinical implications for future research.

Keywords: Sexual relationship; Thematic analysis; Narcissism
\end{abstract}

Received: 17 June 2018, Accepted: 1 October 2018, ePublished: 24 June 2019

\section{Introduction}

Narcissism is considered as one of the oldest concepts in psychology, especially in the psychoanalytic approach. During the past few decades, this concept has been investigated and studied both as a clinical disorder and a non-clinical personality trait. Although many studies have been devoted to narcissism, there are still many areas of uncertainty surrounding its nature and function (16). Some researchers believe that the number of normal people diagnosed with narcissism has increased during the past decades (7). Those people with narcissism suffer from many problems, which are rooted in their needs for admiration, attention, along with their manipulative, selfish, patronizing, and demanding personalities. In addition, they have difficulties regarding recognizing the others' feelings, emotions, needs, and desires and usually start a relationship so that their self-esteem can be strengthened by other people (8). Although a narcissistic person believes in his/her power, he/she needs other people's attention, admiration, and approval as well. In other words, they cannot live without others' attention and admirations. Previous studies show that people with narcissism manage their relationships in order to protect their positive self-view (9-13), which might have some negative consequences for their partners (14-16).

Although narcissism is more frequent in men (17), women are being reported to have more interpersonal problems related to their traits and disorders than men (18). This might be partially because narcissism is not wildly accepted as normal in women and might cause more interpersonal problems. Further, narcissists tend not to value the relationships unless it is for self-serving purposes (19). Narcissism has been connected with sexuality since Ellis first introduced its construct. Since then, the link between narcissism and sexuality has been central to numerous theories of narcissism and sexual behaviors (20-22). Furthermore, narcissistic individuals use their relationships and partners to fulfill their narcissistic needs

(c) 2019 The Author(s); Published by Shahrekord University of Medical Sciences. This is an open-access article distributed under the terms of the Creative Commons Attribution License (http://creativecommons.org/licenses/by/4.0), which permits unrestricted use, distribution, and reproduction in any medium, provided the original work is properly cited. 
(23). Sexuality is not just a physical act, but a social process that is suffused with meaning (24). Despite the strong theoretical connection between narcissism and sex, the body of empirical research that explicitly examines the links between narcissism and sexuality is notably sparse (25). Sexuality is regarded as a fundamental aspect of human experience that is linked to a number of important outcomes. In the right context, sex can foster intimacy and relationship satisfaction $(26,27)$. Narcissists have a heightened sense of sexuality, but they tend to view sex very differently as compared to other people (21).

Although narcissism is a universal concept, some studies indicate that narcissism is widely influenced by the individuals' culture (28). In a cross-cultural study, Ghorbani et al administered the Margolis-Thomas measure of narcissism to both Iranian and American students and observed that unhealthy narcissism is more frequent among Iranian students compared to their American counterparts (29). This is contradictory to the widely accepted beliefs that narcissism is more frequent in a more individualistic society (30). It should be noted that the questionnaire used in the study conducted by Ghorbani et al examined only unhealthy narcissism.

Each culture provides its people with certain methods to satisfy their narcissistic needs. As an example, infidelity and low marital commitment are among the most frequent problems narcissistic people are faced with $(31,32)$. Further, the dual standards of each culture are the factors that determine the frequency of these behaviors. In this regard, Iranian culture has different values and standards for men and women (33). Accordingly, identifying what factors contribute to sexual satisfaction may have important implications for understanding and treating sexual and relationship distress. Reviewing the literature, we noticed that there was a complete absence of a study investigating the perceived sex lives of the wives of narcissist men. Thus, this study sought to investigate women's perception of sex life with narcissist men.

\section{Materials and Methods}

To have a better understanding of the concept, a qualitative study was conducted using a thematic analysis approach. One of the advantages of the thematic approach is that it provides deeper analysis, as well as greater objectivity compared to other data-gathering approaches such as questionnaires (34). All marital counseling centers in Isfahan, which were specialized in couple therapy and personality, were selected (8 centers). Among the clients of these centers, those whose husbands were diagnosed with narcissism were contacted, among whom, 44 couples were selected through a convenience sampling method. Prior to the study, these couples were clinically interviewed once more and were diagnosed and labeled as having narcissism based on DSM-5. The Structured Clinical Interview for DSM-5 (SCID-5) is a semi-structured interview guide for making the major DSM-5 diagnoses and the Personality Disorder Version (SCID-5-PD) is used to evaluate the 10 DSM-5 personality disorders. The researcher also conducted the narcissistic personality inventory (NPI-40). NPI-40 (35) is the most widely used measure of narcissism in social psychological research that is based on DSM-III clinical criteria for narcissistic personality disorder. The inclusion criteria of the research sample were the diagnosis of narcissistic personality in men, the lack of personality or other severe clinical disorders as it interferes in the interview process, the lack of recognition of a personality or a specific clinical disorder in the spouse, an interest in and willingness to volunteer in research and expressing experiences. Moreover, the exclusion criteria included the lack of cooperation in interviews and the completion of the questionnaires. It is worth mentioning that these men's wives had no significant psychological problems and men partially met the criteria of narcissism (i.e., grandiose). The narcissistic men in this study did not meet all criteria for narcissistic personality disorder, but they had several symptoms of narcissism and high scores in the NPI.

After contacting the couples, the women were interviewed and interviews continued until it reached saturation number of 31 couples. Stopping data collection is dependent on 'redundancy' of information or 'saturation'. Redundancy is 'the process of sequentially conducting interviews until all concepts are repeated multiple times without new concepts or themes emerging (36). Moreover, Creswell suggested only 20-30 interviews in qualitative analyses (37), which is consistent with the sample size in this study. Prior to the study, these couples were asked to complete an informed consent form, which was approved by Isfahan University Review Board.

In this study, we were not merely describing the data but were also interested in data analysis. Therefore, thematic analysis was used (38) and performed in six stages. The first stage was performed by the interviewer after each interview and included transcribing the interviews word by word. These transcriptions were then read in the group for a number of times so that the initial coding could emerge. The second stage included the extraction of the primary codes. Therefore, similar codes were grouped into similar classifications. Next, the themes were looked for and the data were classified into similar codes. The fourth stage included reading the data again and specifying each coding system and classification. Data analysis and data gathering (i.e., interviews) continued until the saturation of the data (i.e., when no new data are reported by the participants). In the fifth stage, themes were defined and put into groups by different names. The final stage was the final preparation of the data. Inter-rater reliability was considered throughout the analysis and was more than $76 \%$ (Cohen's kappa coefficient $(\kappa)>76$ ). The differences between the raters were also studied and the codes were examined in the final report. To prevent rater 
bias, interviews were given to another trained analyst and were coded again. This time, the inter-rater reliability was reported to be $70 \%$.

\section{Procedure}

A semi-structured interview was conducted to understand the perceived lives of women living with narcissistic men. These interviews were based on the manual which was recommended by Smith and Osborn (39). The questions in this interview specifically focused on the perceived understanding of women living with narcissistic men. Three initial interviews were used as pilot cases and questions went through some minor changes. Each interview lasted for 45 minutes to 1 hour and was recorded and transcribed word by word for further analysis.

\section{Results}

\section{Descriptive findings}

The average age of the female participants and their husbands were $31.6(\mathrm{SD}=4.68)$ and $33.54(\mathrm{SD}=5.06)$, respectively, and the mean duration of their marriage was 6.51 ( $\mathrm{SD}=3.39)$. A summary of the demographic characteristics of the participants is given in Table 1 .

\section{Qualitative findings: thematic analysis}

During the thematic analysis of the interviews, two main themes regarding the sexual experiences of living with a narcissistic husband were identified including insecure relationship, along with the demanding and one-sided relationship. The first theme contained the lack of sexual intimacy, sexual aggression and unwanted sex, and low commitment. The other main theme encompassed two sub-themes of unrealistic and excessive sexual expectations of the spouse and their relationship and the lack of responsibility and cooperation of the husband. These themes are provided in Table 2 .

Main theme 1: insecure relationship

All women reported that they did not feel secure in their

Table 1. Demographic characteristics of the participants

\begin{tabular}{lcc}
\hline Characteristic & Men & Women \\
\hline Education, No. (\%) & $7(23)$ & $3(10)$ \\
High school & $8(25)$ & $7(23)$ \\
Diploma & $8(25)$ & $12(39)$ \\
Bachelor & $7(23)$ & $6(19)$ \\
Associate Master & $1(3)$ & $3(10)$ \\
Doctoral & & \\
Ethnicity, N0. (\%) & $16(52)$ & $18(58)$ \\
Fars & $8(26)$ & $3(10)$ \\
Lor & $7(23)$ & \\
Tork & & $7(23)$ \\
Prior treatment, (No. \%) & $24(77)$ & $4(13)$ \\
None & $3(10)$ & $31.6 \pm 4.68$ \\
Premarital education & $4(13)$ & \\
Couple therapy & $33.54 \pm 5.06$ & \\
Age, mean \pm SD & &
\end{tabular}

relationships, but felt as if they received no emotional support while experiencing violence and unwanted sex in their relationships. This was a representation of a general dissatisfaction, which was reflected throughout their relationship.

\section{Sub-theme 1: lack of sexual intimacy}

This theme was one of the most important characteristics of the relationships with a narcissistic husband. Most participants (83\%) started the interview as if they had not experienced sexual intimacy in their relationship. These women indicated that their husbands were not paying enough attention or failed to react appropriately to their feelings. Samira, a 29-year old woman, mentioned that "... He just enjoyed himself while I did not experience sexual pleasure... after having sex, he completely neglected me. He ignores my own needs." In addition, Zari (27 years old) indicated that "My husband does not even think I have feelings as well. He does not know that I have a need for sex." Another woman named Bahar (35 years old) claimed that "We have no intimate sex...our sex is unrelenting". These statements reveal a lack of sexual intimacy in participants' relationships.

\section{Sub-theme 2: sexual aggression and unwanted sex}

Almost all participants (93\%) mentioned this theme during the interviews. Generally, women reported that they experienced some form of sexual harassment. Maryam, who was 34 years old, told the interviewer that "He loves forced sex and enjoys more." Further, Nazanin, a 31-yearold woman, stated that "I'm really annoyed during our sexual relationship." Another woman named Sara (29 years old) mentioned that "He is very rough." Overall, women living with narcissistic husbands experienced violence and coercion in their sexual relationships.

\section{Sub-theme 3: low commitment}

The majority of the participants (74\%) of this study reported that they are afraid of the infidelity of their spouses and their husbands did not have enough commitment. Nazanin (32 years old) asserted that "I'm really scared. He is not committed. Only his very need is important." Furthermore, Sara, a 29-year-old woman indicated that "She dates other women." Moreover, Mari (28 years old) declared that "The infidelity is not so bad for him." It is

Table 2. Themes and subthemes of the wives' perception of sexual relationship with a narcissistic man

\begin{tabular}{ll}
\hline Themes & Sub-themes \\
\hline \multirow{3}{*}{ Insecure relationship } & $\begin{array}{l}\text { Lack of sexual intimacy } \\
\text { Sexual aggression and unwanted sex } \\
\text { Low commitment }\end{array}$ \\
& $\begin{array}{l}\text { Unrealistic and excessive sexual } \\
\text { expectations of the spouse and the } \\
\text { relationship } \\
\text { One-sided and demanding } \\
\text { relationship }\end{array}$ \\
& Lack of responsibility and cooperation \\
\hline
\end{tabular}


worth mentioning that many participants in this study insisted that their husbands' definitions of infidelity were quite different.

Main theme 2: one-sided and demanding relationship Most participants (80\%) in this study emphasized that they were stuck in a one-sided relationship and gave more than what received. Additionally, most of these women felt like they were the losers in their sexual relationships. They permanently found themselves to be the second most important person in their husbands' lives.

\section{Sub-theme 1: the unrealistic and excessive sexual expectations of the spouse and relationship}

They reported that their husbands had unrealistic and excessive expectations in their sexual relationships and these expectations were sometimes unclear. They further mentioned that they always had to pay attention to their husbands, which made them tired of the need for constant attention. Mina, a 30-year-old woman, indicated that "Everything should be as he likes. He always wants me to satisfy all his needs." Another woman called Elham (33 years old) asserted that "I must always admire him... He thinks he's an exceptional sexual partner." In addition, Zari (39 years old) stated that "His needs never end ... he's more concerned with his performance than me... He expects me to be "on call" and satisfy his sexual desire".

Sub-theme 2: lack of responsibility and cooperation of husband According to the claims of the wives of narcissistic husbands $(83 \%)$, their husbands were not so cooperative during sexual relationship, whereas they only focused on their own needs. In addition, they did not accept the responsibility for their wrong behaviors. Nazi, a 33-yearold woman, expressed that "Our sex is completely one-sided." Akram, a 32-year-old woman who married a narcissistic man, also indicated that "He never seems to accept what he does wrong". Zari (39 years old) mentioned that "He has never cared about my satisfaction. When I want him to do something, he never listens to me."

\section{Discussion}

Narcissism is considered as a personality style characterized by tendencies towards exploiting the others, a general lack of empathy for others, a pervasive pattern of grandiosity, and an excessive need for admiration (8). Although there have been many studies on narcissism, there are still many unanswered questions about it. Many studies demonstrated that individuals diagnosed with narcissism are faced with many interpersonal problems $(10,40)$. In addition, other studies showed that these difficulties are more severe among women compared to men $(14,18)$. However, men are encountered with some difficulties as well. The purpose of this study was to investigate the sexual perception of women living with narcissistic men in their marital lives. Accordingly, a qualitative study was conducted and the obtained data were analyzed using thematic analysis after a semi-structured interview.

Two main themes of the present study included insecure sexual relationship and one-sided and demanding sexual relationship. The results of this study are in line with DSM5 model for narcissistic personality disorders, in which it is believed that narcissistic individuals are faced with numerous problems because of their needs for approval and inattention to others. Narcissists consider sexuality more in terms of power, influence, and as something daring, in contrast to people with low narcissistic qualities who associate sex more with caring and love. However, as one's culture influences all aspects of life, including marital and sexual relationships, these problems cannot be all contributed to the narcissistic aspect of a person's life. It seems that the male-dominant culture of the Iranian society provides men with abundant means and opportunities to satisfy their narcissistic needs, including an androcentrism society, dual standards, and a law system, which is mostly on men's side (33).

\section{Insecure Relationship}

In this regard, the results revealed that the wives of narcissist men did not find their sexual relationships secure enough (i.e., they felt the lack of intimacy and received no emotional support and commitment), which is in accordance with the findings reported by Levy et al (41). They believed that unhealthy attachment is a characteristic of personality disorders. Several studies demonstrated that there is a positive relationship between unhealthy attachments and narcissism $(42,43)$. Sexual problems in individuals with insecure attachment style were highlighted in many studies as well (44-47).

Some researchers maintained that narcissism can be an indicator of sexual aggression (20). In other words, narcissists respond more aggressively to interpersonal rejections across a variety of domains (48-50), a response that may be generalizable to sexual rejections. Possibly, due to their especially great need for positive regard and admiration $(51,52)$, narcissists appear particularly oriented towards sexual relationships (53), an orientation that provides greater opportunities for experiencing the types of rejections that could lead to sexual aggression. The agency model of narcissism suggests that self-regulation, approached orientation, and the extraversion of narcissists make them more inclined towards dating and this leads to less commitment (9).

The findings of this study are consistent with the findings reported by Campbell, in which the authors believed that narcissistic individuals are not looking for caring relationships. They are not able to put themselves in other people's shoes and cannot make contact based on empathy, even though they are not aware of it (54). According to the agency model introduced by Campbell 
et al, people with narcissistic personality disorder are more focused on agentic traits than the communal ones (9). Based on wives' narrations in this study, men think their wives should fulfill their needs and consider it as their wives' duty. In addition, narcissist men with these cultural beliefs cannot tolerate disappointment or rejection. They frequently react negatively when their wives do not give them what they want as they wish to.

To adjust their lost self-esteem, individuals with narcissistic personality might threaten their wives or might seek their lost self-esteem in another relationship. Several empirical studies highlighted significant associations between narcissistic personality traits and more unrestricted socio-sexuality orientations (21, 55). Individuals with more unrestricted socio-sexuality orientations are comfortable with engaging in sex with no commitment or intimacy. Foster et al demonstrated that narcissistic individuals prefer less committed and less restricted sexual relationships because they are self-focused and prefer higher individuality rather than intimacy in their sexual encounters (21).

\section{One-sided and demanding relationship}

According to the reports by the participants regarding the second major theme, women found that their sexual relationships with their husbands were one-sided and demanding. The theme in this category (which is unrealistic with excessive expectations of the husbands and the lack of responsibility and cooperation on husbands' part) makes the sexual relationship as one-sided in women's eyes. Campbell and Foster believed that narcissistic people are involved in game-playing love, which demands them to be practical and selfish in their romantic relationships (11). In addition, according to the agency model of narcissism, a model of the action of narcissistic individuals is about status, dominance, and autonomy (56). These characteristics are in conflict with cooperation and accepting responsibilities in any domain. Further, Back et al stated that interpersonal problems of a narcissistic person are evident at his/her home and in the family more than any other places. Their family members are not only responsible for satisfying their numerous needs but also must predict what their needs might be (57). It seems that this process is also true in their sexual relationship. Millon et al presumed that a narcissistic person expects others to understand his/her wisdom rather than trying to communicate with others based on empathy and love (58).

Women also mentioned high expectations of their husbands as another characteristic of sex life with a person diagnosed with narcissism. Notably, some cultural beliefs and values might aggravate these ideas. It is understood that narcissist men use cultural prototypes, which more often approve of their behaviors. According to Farwell and Wohlwend-Lloyd and Twenge et al, narcissistic individuals have great expectations of themselves $(7,59)$. In this study, women complained about their husbands' permanent expectations respecting their constant attention to their spouse in their sexual relationships. Consistent with the agency model (21) and probably what we expected, narcissists tended to score higher and lower on agentic and communal sexuality, respectively. This finding suggests that, like everything else in their lives, narcissists view sex as being "all about me."

Similar to other studies, this study suffers from a number of limitations. The generalization of the data might not be possible to other samples and communities because of the limited number of participants. Another limitation is the fact that sexual relationship is quite multi-faceted. Therefore, understanding the exact reasons or consequences of a phenomenon might not be an easy job. Thus, some of the findings of this study might not be directly related to the narcissistic personality of the individuals. In addition, the sample was selected through a convenience sampling technique, which might make it difficult to generalize the data to other groups. Further, the type of vulnerability and sexual narcissism were not investigated in this study, which can be examined in similar future research.

However, there are potential clinical implications for this study. The wives' perception of sexual relationship with a narcissistic man can be the basis for sex therapy in these couples. Drawing on the results of this study, therapists can better understand the subjective experience of spouses and thus choose the type of requisite interventions and prioritize them based on the perceptions of their spouses. Moreover, therapists can incorporate cultural considerations into their work and professional expertise. The results of this research are also important for premarital counselors to help their clients find out how the sexual experience with a narcissistic man is and what problems they may encounter in this regard.

\section{Conclusion}

Narcissism is believed to be a complex and multidimensional personality trait and predicts impaired functioning across a variety of marital life domains. Based on the findings of the current study, women were found to face numerous challenges in their sexual relationships when living with a narcissistic person. The results of this study further suggest that sexual relationships are influenced by the narcissistic personality of the husband and, in return, his narcissism is affected by numerous cultural factors. Importantly, it seems that Iranian culture provides men with more opportunities to satisfy their narcissistic needs and desires.

Conflict of interests

None.

Acknowledgments

This research was supported by University of Isfahan and approved 
on January 3rd, 2014 with the project number of 1514036 and the ethical permission code of IR.UI.REC.1397.089. We thank our colleagues who provided insights and expertise that greatly assisted the study.

\section{References}

1. Miller JD, Campbell WK, Pilkonis PA. Narcissistic personality disorder: relations with distress and functional impairment. Compr Psychiatry. 2007;48(2):170-7. doi: 10.1016/j. comppsych.2006.10.003.

2. Miller JD, Widiger TA, Campbell WK. Narcissistic personality disorder and the DSM-V. J Abnorm Psychol. 2010;119(4):6409. doi: 10.1037/a0019529.

3. Pincus AL, Lukowitsky MR. Pathological narcissism and narcissistic personality disorder. Annu Rev Clin Psychol. 2010;6:421-46. doi: 10.1146/annurev. clinpsy.121208.131215.

4. Skodol AE, Bender DS, Morey LC. Narcissistic personality disorder in DSM-5. Personal Disord. 2014;5(4):422-7. doi: 10.1037/per0000023.

5. Miller JD, Lynam DR, Hyatt CS, Campbell WK. Controversies in Narcissism. Annu Rev Clin Psychol. 2017;13:291-315. doi: 10.1146/annurev-clinpsy-032816-045244.

6. Sandler J, Person ES, Fonagy P. On narcissism: An introduction. Freud's" On Narcissism. Routledge; 2018. p. 3-32.

7. Twenge JM, Konrath S, Foster JD, Campbell WK, Bushman BJ. Egos inflating over time: a cross-temporal meta-analysis of the Narcissistic Personality Inventory. J Pers. 2008;76(4):875-902; discussion 3-28. doi: 10.1111/j.1467-6494.2008.00507.x.

8. American Psychological Association (APA). Diagnostic and statistical manual of mental disorders. 5th ed. APA; 2013.

9. Campbell WK, Brunell AB, Finkel EJ. Narcissism, interpersonal self- regulation, and romantic relationships: An Agency Model Approach. In: Finkel EJ, Vohs KD, eds. Self and Relationships: Connecting Intrapersonal and Interpersonal Processes. The Guilford Press; 2006. p.57-83.

10. Campbell WK, Campbell SM. On the self-regulatory dynamics created by the peculiar benefits and costs of narcissism: A contextual reinforcement model and examination of leadership. Self Identity. 2009;8(2-3):214-32. doi: $10.1080 / 15298860802505129$.

11. Campbell WK, Foster CA. Narcissism and commitment in romantic relationships: An investment model analysis. Pers Soc Psychol Bull. 2002;28(4):484-95. doi: $10.1177 / 0146167202287006$.

12. Campbell WK, Rudich EA, Sedikides C. Narcissism, selfesteem, and the positivity of self-views: Two portraits of self-love. Pers Soc Psychol Bull. 2002;28(3):358-68. doi: $10.1177 / 0146167202286007$.

13. Sedikides C, Campbell WK. Narcissistic force meets systemic resistance: The energy clash model. Perspect Psychol Sci. 2017;12(3):400-21. doi: 10.1177/1745691617692105.

14. Lamkin J, Lavner JA, Shaffer A. Narcissism and observed communication in couples. Pers Individ Dif. 2017;105:224-8. doi: 10.1016/j.paid.2016.09.046.

15. Tortoriello GK, Hart W. Modeling the interplay between narcissism, relational motives, and jealousy-induced responses to infidelity threat. J Soc Pers Relatsh. 2019;36(7):2156-79. doi: $10.1177 / 0265407518783096$.

16. Gewirtz-Meydan A. Why do narcissistic individuals engage in sex? Exploring sexual motives as a mediator for sexual satisfaction and function. Pers Individ Dif. 2017;105:7-13. doi: 10.1016/j.paid.2016.09.009.

17. Grijalva E, Newman DA, Tay L, Donnellan MB, Harms PD, Robins RW, et al. Gender differences in narcissism: a metaanalytic review. Psychol Bull. 2015;141(2):261-310. doi: 10.1037/a0038231.

18. Lavner JA, Lamkin J, Miller JD, Campbell WK, Karney BR.
Narcissism and newlywed marriage: Partner characteristics and marital trajectories. Personal Disord. 2016;7(2):169-79. doi: 10.1037/per0000137.

19. Casale S, Rugai L, Fioravanti G, Puccetti C. Narcissism and authentic self: An unfeasible marriage? Pers Individ Dif. 2018;135:131-6. doi: 10.1016/j.paid.2018.07.008.

20. Baumeister RF, Catanese KR, Wallace HM. Conquest by force: A narcissistic reactance theory of rape and sexual coercion. Rev Gen Psychol. 2002;6(1):92-135. doi: 10.1037//10892680.6.1.92.

21. Foster JD, Shrira I, Campbell WK. Theoretical models of narcissism, sexuality, and relationship commitment. J Soc Pers Relatsh. 2006;23(3):367-86.

22. Freud S. On narcissism: an introduction. In: Strachey J, ed. The standard edition of the complete psychological works of Sigmund Freud. London : The Hogarth Press; 1914.

23. Beck AT, Davis DD, Freeman A. Cognitive therapy of personality disorders. Guilford Publications; 2015.

24. Baumeister RF, Tice DM. The social dimension of sex. Boston: Allyn and Bacon; 2001.

25. Widman L, McNulty JK. Narcissism and sexuality. In: Campbell WK, Miller JD, eds. The handbook of narcissism and narcissistic personality disorder: Theoretical approaches, empirical findings, and treatments. Hoboken, NJ, US: John Wiley \& Sons Inc; 2011:351-9.

26. Little KC, McNulty JK, Russell VM. Sex buffers intimates against the negative implications of attachment insecurity. Pers Soc Psychol Bull. 2010;36(4):484-98. doi: 10.1177/0146167209352494.

27. Meltzer AL, McNulty JK. Body image and marital satisfaction: evidence for the mediating role of sexual frequency and sexual satisfaction. J Fam Psychol. 2010;24(2):156-64. doi: 10.1037/ a0019063.

28. Twenge JM, Campbell WK. Personality psychology: understanding yourself and others. Boston: Pearson; 2017.

29. Ghorbani N, Watson PJ, Hamzavy F, Weathington BL. Selfknowledge and narcissism in Iranians: Relationships with empathy and self-esteem. Curr Psychol. 2010;29(2):135-43. doi: 10.1007/s12144-010-9079-5.

30. Heine SJ, Hamamura T. In search of East Asian selfenhancement. Pers Soc Psychol Rev. 2007;11(1):4-27. doi: 10.1177/1088868306294587.

31. Keith Campbell W, Miller JD, Buffardi LE. The United States and the "Culture of Narcissism" An examination of perceptions of national character. Soc Psychol Personal Sci. 2010;1(3):222-9. doi: 10.1177/1948550610366878.

32. McNulty JK, Widman L. Sexual narcissism and infidelity in early marriage. Arch Sex Behav. 2014;43(7):1315-25. doi: 10.1007/s10508-014-0282-6.

33. Parnian $\mathrm{H}$. The sexual rights of women and homosexuals in Iran. In: Seidman S, Fischer N, Meeks C, eds. Handbook of the new sexuality studies. New York, NY: Routledge; 2006. p.348-52

34. Smith JA. Evaluating the contribution of interpretative phenomenological analysis. Health Psychol Rev. 2011;5(1):927. doi: 10.1080/17437199.2010.510659.

35. Safarinia M, Shaghaghi F, Maleki B. Preliminary Study of Psychometric Properties of Narcissistic Personality Questionnaire. Journal of Clinical Psychology Studies. 2012;8:61-74.

36. Trotter RT, 2nd. Qualitative research sample design and sample size: resolving and unresolved issues and inferential imperatives. Prev Med. 2012;55(5):398-400. doi: 10.1016/j. ypmed.2012.07.003.

37. Creswell JW, Creswell JD. Research design: qualitative, quantitative, and mixed methods approaches. Sage Publications; 2017.

38. Braun V, Clarke V. Using thematic analysis in 
psychology. Qual Res Psychol. 2006;3(2):77-101. doi: 10.1191/1478088706qp063oa.

39. Smith JA, Osborn M. Interpretative phenomenological analysis. Wiley Online Library; 2004.

40. Peterson JL, DeHart T. In defense of self-love: An observational study on narcissists' negative behavior during romantic relationship conflict. Self Identity. 2014;13(4):477-90. doi: 10.1080/15298868.2013.868368.

41. Levy KN, Johnson BN, Scala JW, Temes CM, Clouthier TL. An attachment theoretical framework for understanding personality disorders: Developmental, neuroscience, and psychotherapeutic considerations. Psihol Teme. 2015;24(1):91-112.

42. Ahmadi V, Ahmadi S, Honarmand MM, Zargar Y, Arshadi N, Mirshekar S. The Relationships Between Attachment Styles and Narcissism among Students of Shahid Chamran University in Iran. Procedia Soc Behav Sci. 2013;84:215-8. doi: 10.1016/j. sbspro.2013.06.537.

43. Lyddon WJ, Sherry A. Developmental personality styles: An attachment theory conceptualization of personality disorders. J Couns Dev. 2001;79(4):405-14. doi: 10.1002/j.15566676.2001.tb01987.x.

44. Nia AS, Salari P, Sharifi N, Nooghani HJ. Effect of Attachment Styles to Parents on Sexual Dysfunction Domains of Married Women. Electron Physician. 2017;9(1):3605-10. doi: $10.19082 / 3605$

45. Tracy JL, Shaver PR, Albino AW, Cooper ML. Attachment styles and adolescent sexuality. In: Florsheim P, ed. Adolescent romantic relations and sexual behavior: Theory, research, and practical implications. Mahwah, NJ: Lawrence Erlbaum Associates Publishers; 2003:137-59.

46. Stefanou C, McCabe MP. Adult attachment and sexual functioning: A review of past research. J Sex Med. 2012;9(10):2499-507. doi: 10.1111/j.1743$6109.2012 .02843 . x$.

47. Mark KP, Vowels LM, Murray SH. The impact of attachment style on sexual satisfaction and sexual desire in a sexually diverse sample. J Sex Marital Ther. 2018;44(5):450-8. doi: 10.1080/0092623x.2017.1405310.

48. Baumeister RF, Bushman BJ, Campbell WK. Self-esteem, narcissism, and aggression: Does violence result from low self-esteem or from threatened egotism? Curr Dir Psychol Sci. 2000;9(1):26-9. doi: 10.1111/1467-8721.00053.

49. Bushman BJ, Bonacci AM, van Dijk M, Baumeister RF. Narcissism, sexual refusal, and aggression: testing a narcissistic reactance model of sexual coercion. J Pers Soc Psychol. 2003;84(5):1027-40.

50. Konrath S, Bushman BJ, Campbell WK. Attenuating the link between threatened egotism and aggression. Psychol Sci. 2006;17(11):995-1001. doi: 10.1111/j.14679280.2006.01818.x.

51. Morf CC, Rhodewalt F. Unraveling the paradoxes of narcissism: A dynamic self-regulatory processing model. Psychol Inq. 2001;12(4):177-96. doi: 10.1207/S15327965PLI1204_1.

52. Raskin R, Novacek J, Hogan R. Narcissistic self-esteem management. J Pers Soc Psychol. 1991;60(6):911-8. doi: 10.1037/0022-3514.60.6.911.

53. Schmitt DP, Alcalay L, Allik J, Alves ICB, Anderson CA, Angelini AL, et al. Narcissism and the strategic pursuit of short-term mating: Universal links across 11 world regions of the international sexuality description project-2. Psihol Teme. 2017;26(1):89-137. doi: 10.31820/pt.26.1.5.

54. Campbell WK. Narcissism and romantic attraction. J Pers Soc Psychol. 1999;77(6):1254-70. doi: 10.1037/00223514.77.6.1254.

55. Jonason PK, Li NP, Webster GD, Schmitt DP. The dark triad: Facilitating a short-term mating strategy in men. Eur J Pers. 2009;23(1):5-18. doi: 10.1002/per.698.

56. Campbell WK, Foster JD. The narcissistic self: Background, an extended agency model, and ongoing controversies. In: Sedikides C, Spencer SJ, eds. Frontiers of social psychology. The Self. New York, NY: Psychology Press; 2007. p.115-38.

57. Back MD, Kufner AC, Dufner M, Gerlach TM, Rauthmann JF, Denissen JJ. Narcissistic admiration and rivalry: disentangling the bright and dark sides of narcissism. J Pers Soc Psychol. 2013;105(6):1013-37. doi: 10.1037/a0034431.

58. Millon T, Grossman S, Millon C, Meagher S, Ramnath R. Personality disorders in modem life. Hoboken, NJ: John Wiley; 2004.

59. Farwell L, Wohlwend-Lloyd R. Narcissistic processes: optimistic expectations, favorable self-evaluations, and selfenhancing attributions. J Pers. 1998;66(1):65-83. 